\title{
Preexisting pancreatic acinar cells contribute to acinar cell, but not islet $\beta$ cell, regeneration
}

\author{
Biva M. Desai, ${ }^{1}$ Jennifer Oliver-Krasinski, ${ }^{1}$ Diva D. De Leon,, ${ }^{1,2}$ Cyrus Farzad, ${ }^{1}$ \\ Nankang Hong, ${ }^{1}$ Steven D. Leach, ${ }^{3}$ and Doris A. Stoffers ${ }^{1}$
}

\begin{abstract}
1Division of Endocrinology, Diabetes, and Metabolism and Institute for Diabetes, Obesity, and Metabolism, University of Pennsylvania School of Medicine, Philadelphia, Pennsylvania, USA. ²Division of Pediatric Endocrinology, Children's Hospital of Philadelphia, Philadelphia, Pennsylvania, USA. ${ }^{3}$ Departments of Surgery and Cell Biology, Johns Hopkins University School of Medicine, Baltimore, Maryland, USA.
\end{abstract}

\begin{abstract}
It has been suggested that pancreatic acinar cells can serve as progenitors for pancreatic islets, a concept with substantial implications for therapeutic efforts to increase insulin-producing $\beta$ cell mass in patients with diabetes. We report what we believe to be the first in vivo lineage tracing approach to determine the plasticity potential of pancreatic acinar cells. We developed an acinar cell-specific inducible Cre recombinase transgenic mouse, which, when mated with a reporter strain and pulsed with tamoxifen, resulted in permanent and specific labeling of acinar cells and their progeny. During various time periods of observation and using several models to provoke injury, we failed to observe any chase of the labeled cells into the endocrine compartment, indicating that acinar cells do not normally transdifferentiate into islet $\beta$ cells in vivo in adult mice. In contrast, we observed a substantial role for replication of preexisting acinar cells in the regeneration of new acinar cells after partial pancreatectomy. These results indicate that mature acinar cells harbor a facultative acinar but not endocrine progenitor capacity.
\end{abstract}

\section{Introduction}

The pancreas is a complex organ composed of endocrine and exocrine compartments. The exocrine compartment, comprising the large majority of pancreas, contains the acinar cells, which produce digestive enzymes, and the branching ductal system, which delivers these enzymes to the duodenum. The endocrine compartment, which constitutes just $1 \%-2 \%$ of the adult pancreas, produces islet hormones such as insulin and glucagon that are critically required for the maintenance of normal glucose homeostasis.

The plasticity of the adult pancreas is a subject of substantial clinical relevance. Diabetes, which is regarded as a global epidemic, is caused by impaired islet compensation in the setting of insulin resistance. For both type 1 and type 2 diabetes, inadequate functional $\beta$ cell mass is a critical component of disease pathophysiology (reviewed in ref. 1). Although islet transplantation has demonstrated potential to cure type 1 diabetes, therapy is limited by the toxicity of immune suppression and by the grossly inadequate supply of donor islets (reviewed in refs. 2, 3). Therefore, alternative sources of $\beta$ cell replacement are being intensively sought, including directed differentiation from embryonic stem cells (reviewed in ref. 4) and transdifferentiation from other cell types such as hepatocytes (5). Of even greater impact would be a strategy that promotes in vivo transdifferentiation of $\beta$ cells, thereby obviating the need for immune suppression to prevent rejection of foreign cells.

Acinar cells are an attractive alternative source of islet progenitors because they are plentiful within the pancreas and they share with islet $\beta$ cells a common pancreas duodenal homeobox-1expressing (PDX-1-expressing) progenitor during embryonic development. It has previously been reported that acinar cells have considerable plasticity and can differentiate into cells of ductal

Nonstandard abbreviations used: Ex-4, exendin-4; PDX-1, pancreas duodenal homeobox-1; Ppx, partial pancreatectomy; TAM, tamoxifen.

Conflict of interest: The authors have declared that no conflict of interest exists. Citation for this article: J. Clin. Invest. 117:971-977 (2007). doi:10.1172/JCI29988.
$(6-8)$ and hepatic lineages $(9,10)$ in culture. Based on coexpression of insulin and amylase during regeneration in several models, acinar-to-islet transdifferentiation has also been suggested (11-13). Furthermore, the pancreatic acinar AR42J tumor cell line can be induced by various combinations of activin A, betacellulin, HGF, and glucagon-like peptide- 1 to transdifferentiate into endocrine $\beta$-like cells that express insulin $(9,14,15)$. However, many of these studies failed to formally exclude nonacinar sources for $\beta$ cell neogenesis, based on a limited number of available markers and an inability to rigorously trace the fate of individual cell types. In addition, the potential for these transdifferentiation events to occur in vivo has not been established. With the advent of Cre recombinase-LoxP methodology, currently available lineage tracing approaches now allow for a more rigorous analysis of any proposed transdifferentiation process.

Here, we present the results of what we believe to be the first reported lineage tracing approach to determine the in vivo plasticity potential of acinar cells. An acinar cell-specific inducible Cre recombinase transgenic mouse was developed, which, when mated with a reporter strain and treated with tamoxifen (TAM), resulted in permanent and specific labeling of existing acinar cells and their progeny. During follow-up observation periods of up to 6 weeks and after various interventions to provoke injury and regeneration, we failed to observe labeled cells in the endocrine compartment, indicating that acinar cells do not transdifferentiate into islet $\beta$ cells in vivo. Our data indicate a substantial role for replication of preexisting acinar cells in the regeneration of new acinar cells in adult mice.

\section{Results}

We generated a transgenic mouse in which a TAM-inducible Cre recombinase (CreERT2, which encodes a Cre recombinase [Cre] fused to a mutant estrogen receptor ligand-binding domain [ERT2] that is selectively responsive to TAM) is regulated by the strong and highly acinar cell-specific elastase I promoter (ElastaseCreERT2 
A

Elastase CreERT2 transgene
\begin{tabular}{|c|c|c|c|}
\hline Elas & $\begin{array}{c}\text { RBG } \\
\text { Intron }\end{array}$ & CreERT2 & $\begin{array}{c}\text { RBG } \\
\text { PolyA }\end{array}$ \\
\hline
\end{tabular}
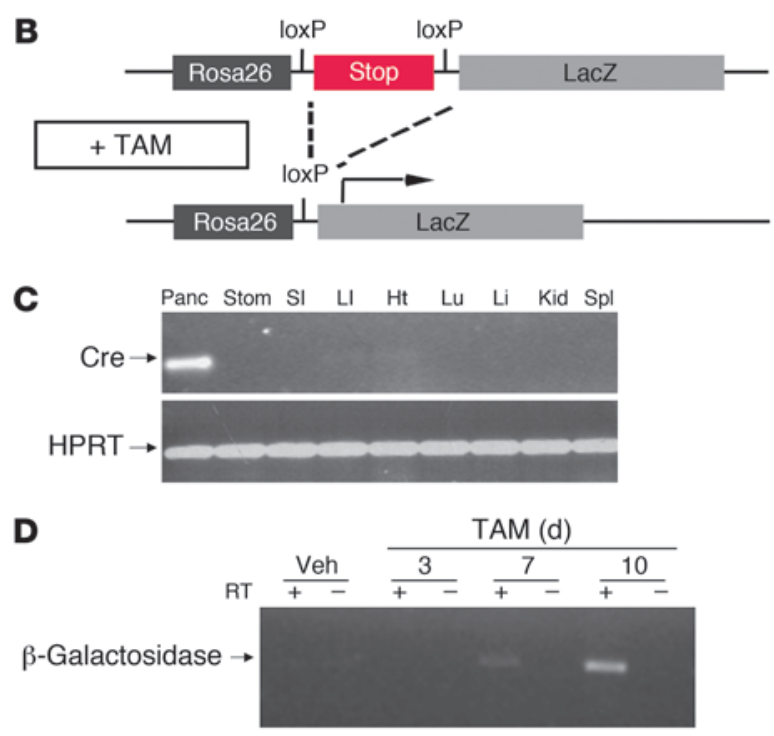

mouse; Figure 1A) (16) in order to facilitate lineage tracing studies and experiments involving inducible and selective gene inactivation in pancreatic acinar cells. Two founders were identified, one of which expressed Cre recombinase, as detected by RT-PCR of pancreatic RNA (Figure 1C). A tissue distribution experiment revealed appropriate restriction of transgene expression to the pancreas (Figure 1C). Notably, no substantial Cre recombinase mRNA expression was detected in the stomach, small or large intestine, heart, lungs, liver, kidney, or spleen. This is consistent with the faithful pancreas-specific expression directed by this promoter fragment previously reported in other transgenic models $(17,18)$.

To determine the efficiency and specificity of Cre-mediated recombination in ElastaseCreERT2 mice, we crossed ElastaseCreERT2 Ty/+ mice with Rosa26r reporter mice (19) to generate ElastaseCreERT2 Ty / Rosa $26 r^{+/-}$mice. Expression of $\beta$-galactosidase was restricted to cells that underwent Cre-mediated excision of an upstream stop cassette in the LacZ-engineered endogenous Rosa locus, as well as the progeny of these cells (Figure 1B). To determine the levels of basal and TAM-induced Cre-mediated recombination, RT-PCR for $\beta$-galactosidase mRNA was carried out using RNA isolated from the pancreata of ElastaseCreERT2 $2^{\mathrm{Tg} /+}$ Rosa $6 r^{+-}$- mice left untreated or treated for 3, 7, or 10 days with TAM. As shown in Figure 1D, no $\beta$-galactosidase mRNA was observed in untreated mice, indicating no detectable basal Cre-mediated recombination in the absence of TAM treatment. Recombination was detected by 7 days of treatment, and higher levels of recombination were observed after 10 days of treatment.

To determine whether transgene expression is restricted to the acinar lineage of the pancreas, ElastaseCreERT2 $2^{\mathrm{Tg} /+}$ mice were initially treated with TAM for 3 days or left untreated. Pancreatic sections were visualized for immunoreactivity associated with Cre recombinase as well as insulin, a key marker of the endocrine pancreatic $\beta$ cell that constitutes the majority of cells in the islets of Langerhans. In the control pancreas, Cre immunoreactivity was localized to the cytoplasm in a subset of pancreatic cells. After 3 days

\section{Figure 1}

CreERT2 expression is restricted to the pancreas of ElastaseCreERT2 transgenic mice. (A) Schematic depiction of the elastase CreERT2 transgene. The transgene contains the $0.5-\mathrm{kb}$ proximal rat elastase I promoter (Elas; gray), the rabbit $\beta$-globin intron (RBG intron; black), the coding region for CreERT2 (white), and the rabbit $\beta$-globin polyadenylation cassette (RBG PolyA; black). (B) Schematic depiction of the Rosa26r reporter locus and the effect of TAM-induced Cre recombinase activity to excise a loxP-flanked stop sequence (dashed lines), thereby allowing transcription of LacZ (i.e., $\beta$-galactosidase; arrow). (C) Tissue distribution of Cre recombinase expression, determined by RT-PCR for Cre recombinase mRNA using total RNA isolated from the designated tissues of 16-week-old transgenic mice. Panc, pancreas; Stom, stomach; SI, small intestine; LI, large intestine; Ht, heart; Lu, lung; Li, liver; Kid, kidney; Spl, spleen. (D) Time course indicating low basal Cre-mediated recombination and high inducibility by TAM administration. RT-PCR for $\beta$-galactosidase mRNA carried out using RNA isolated from pancreata of 16-week-old transgenic mice left untreatd or after 3,7 , or 10 days of TAM treatment.

of TAM treatment, nuclear Cre was observed in a mosaic pattern that was restricted to the exocrine portion of the pancreas (data not shown). Cre recombinase expression was not observed in islets of the endocrine pancreas. After 21 days of TAM treatment delivered by subcutaneous continuous delivery pellet, $\beta$-galactosidase was visualized in approximately $30 \%$ of acinar cells in a patchy but somewhat lobular pattern, reflecting the mosaic expression pattern of Cre recombinase (Figure 2, B-D), as has been frequently observed with other Cre recombinase driver lines (20). In the absence of TAM administration, very few acinar cells expressed $\beta$-galactosidase, indicating tight regulation of Cre activity by TAM (Figure 2A). In TAM-treated mice, no $\beta$-galactosidase-positive cells were detected within islets at any time point. Furthermore, double staining with biotinylated dolichos biflorus agglutinin (DBA) lectin (which selectively recognizes ductal epithelial cells; refs. 21, 22, and data not shown) or with ductal-specific cytokeratin 19 showed complete absence of labeling in all classes of ductal epithelial cells, including the specialized centroacinar cell (Figure 2, E-N). Thus, the elastase CreERT2 transgene is expressed only in the exocrine pancreas, and induction of recombinase activity in adult mice appears to induce loxP rearrangement selectively in acinar cells.

Once characterized, ElastaseCreERT2 ${ }^{\mathrm{Tg} /}{ }^{+} \mathrm{Rosa}_{2} 6 \mathrm{r}^{+/-}$mice were used in lineage tracing studies to determine the transdifferentiation potential of acinar cells in vivo. In control, sham-operated mice, the islet lineage was not labeled during observation periods of up to 6 weeks after the completion of TAM administration (Figure 3), indicating that adult acinar cells do not serve as islet progenitor cells during normal pancreas function in mice. To determine whether acinar-to-islet transdifferentiation occurs under conditions in which islet regeneration is provoked, we performed $70 \%-80 \%$ partial pancreatectomy (Ppx) or sham operation on ElastaseCreERT2 ${ }^{\mathrm{Tg} /}{ }^{+} \mathrm{Rosa}\left(6 \mathrm{r}^{+/-}\right.$mice and then treated them with either vehicle or exendin- 4 (Ex-4) to further promote $\beta$ cell regeneration (23). We confirmed $\beta$ cell mass regeneration by quantitation at 1 month after surgery, when $\beta$ cell mass in the Ppx remnant had 

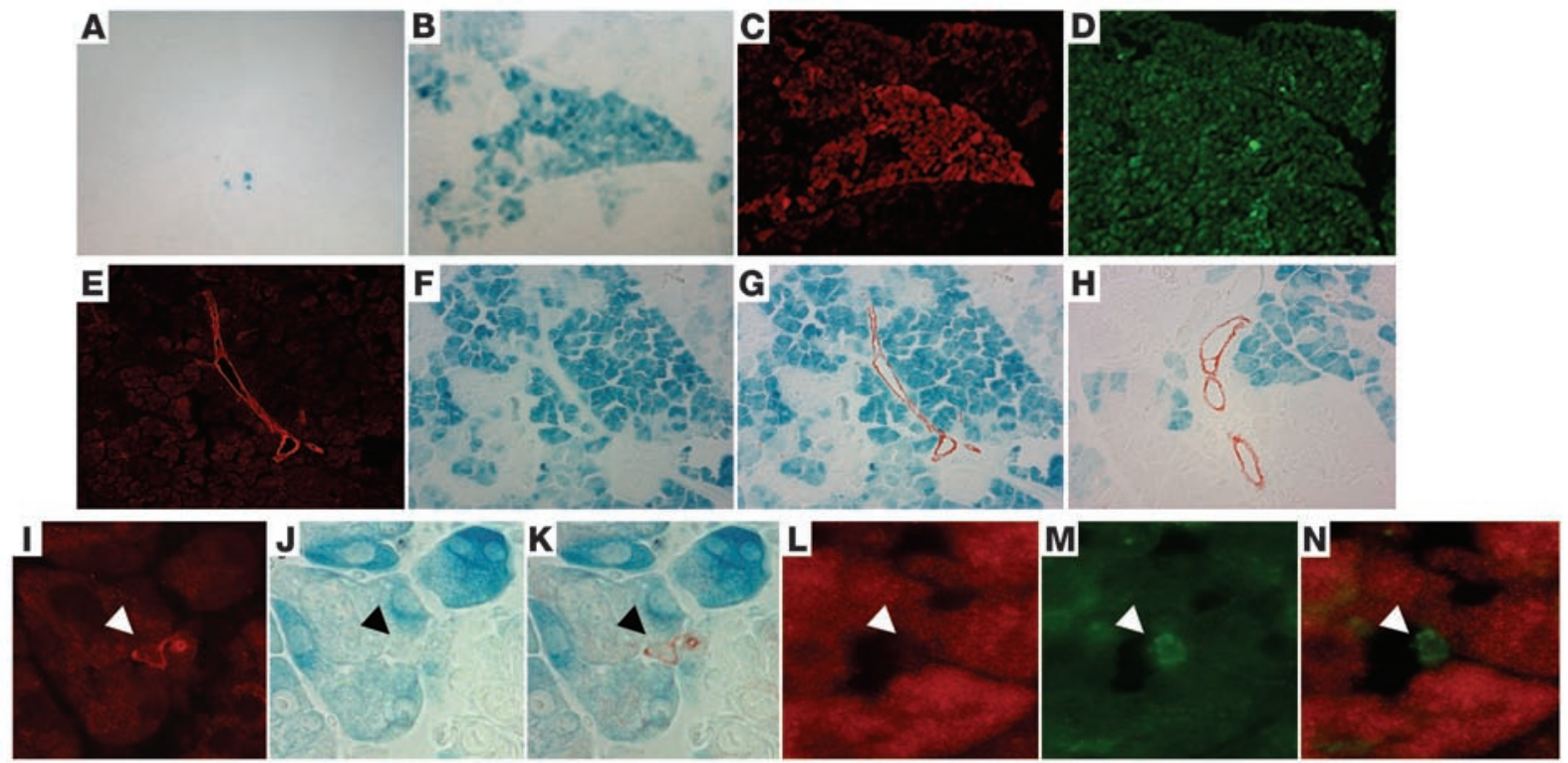

Figure 2

Cre-mediated recombination is restricted to the acinar cell lineage in TAM-treated ElastaseCreERT2Tg/+Rosa26r+/- mice. ElastaseCreERT2 mouse pancreata from 16-week-old animals stained with X-gal (A, B, F-H, J, and K), anti-Cre recombinase antiserum (red stain; C, L, and N), anti-amylase (green stain; D), or anti-cytokeratin 19 (red stain in $\mathbf{E}, \mathbf{G}-\mathbf{I}$, and $\mathbf{K}$; green stain in $\mathbf{M}$ and $\mathbf{N}$ ) without TAM treatment (A) and after 21 days of TAM treatment (B-N). Arrowheads indicate centroacinar cells identified by cytokeratin-19 immunoreactivity (I and $\mathbf{M})$ but no $\beta$-galactosidase activity $(\mathbf{J})$ or Cre recombinase immunoreactivity (L). Original magnification, $\times 200(\mathbf{A}-\mathbf{H}) ; \times 400(\mathbf{I}-\mathbf{K}) ; \times 1,000(\mathbf{L}-\mathbf{N})$.

recovered to $73 \%$ that of the sham-operated group ( $P=$ NS; Figure $4 \mathrm{~B})$, indicating a robust regeneration of $\beta$ cell mass similar to our previous observations in CD1 mice (24). The exocrine portion of the pancreatic remnant only partially recovered, as indicated by reduced wet weight compared with the pancreata of sham-operated controls (Figure 4C). The biologic efficacy of Ex-4 was demonstrated by improved glucose tolerance at 2 weeks in Ex-4- versus vehicle-treated Ppx mice (Figure 4A).

At no time did we observe $\beta$-galactosidase labeling within pancreatic islets, indicating that acinar cells do not serve as islet progenitors during regeneration after Ppx. Furthermore, Ex-4 does not promote acinar-to-islet transdifferentiation in vivo, in contrast to its ability to promote transdifferentiation of acinar tumor AR42J cells into insulin-expressing $\beta$-like cells in culture (14). Similarly, no $\beta$-galactosidase-labeled ductal epithelial cells were observed in vivo, in contrast to our previous observations of acinar-to-ductal transdifferentiation when TAM-treated ElastaseCreERT2 $2^{\mathrm{Tg}}{ }^{+} \mathrm{Rosa}_{2} \mathrm{Gr}^{+/-}$ acini are maintained in culture in the presence of TGF- $\alpha$ (8). Taken together, these data indicate that acinar cells may have limited transdifferentiation potential in vivo.

In contrast, our present studies clearly indicate that preexisting acinar cells serve as progenitors for newly formed acinar cells during regeneration, similar to recent reports of $\beta$ cell replication as the primary mechanism for postnatal $\beta$ cell replacement and during adult islet regeneration $(25,26)$. In order to distinguish between preexisting and newly generated cells, BrdU was administered for 1 week beginning immediately after surgery and continuing until tissue harvest. BrdU was found to be incorporated into patches of acinar cells distributed throughout the acinar parenchyma, but not restricted to individual lobes (Figure 5A). We observed BrdU incorporation into $\beta$-galactosidase-positive and -negative cells, indicating that $\beta$-galactosidase-expressing cells contribute to acinar cell replacement (Figure 5B). To determine whether an unlabeled progenitor cell type, as opposed to differentiated acinar cells, markedly contributes to the formation of new acinar cells, we assessed the extent of $\beta$-galactosidase activity in these areas of high proliferation. If an unlabeled progenitor cell type contributes to acinar cell regeneration, then a decrease in the percentage of cells expressing the $\beta$-galactosidase label should be observed, such that proliferative areas are those with low proportions of cells expressing $\beta$-galactosidase. On the other hand, if acinar cell regeneration occurs primarily through replication of preexisting acinar cells, no decrease in labeling would be expected. In fact, we observed that high levels of BrdU incorporation occurred with equal frequency in areas of high, medium, and low $\beta$-galactosidase expression (Figure 5C), arguing against a contribution from a $\beta$-galactosidase-negative progenitor cell type. Because the specialized centroacinar cell is not labeled in this model, the results also argue against a role for centroacinar cells as progenitors for acinar cell regeneration after Ppx. In agreement with previous studies demonstrating the importance of postnatal $\beta$ cell replication $(25,26)$, we observed robust incorporation of BrdU within regenerating islets after Ppx (Figure 5D).

Finally, we examined acinar cell transdifferentiation potential in 2 other models of pancreatic injury in order to determine whether the ability of acinar cells to serve as islet progenitors is model specific. Pancreatitis was induced by a 2-day course of cerulein injections that resulted in marked exocrine atrophy and inflammatory infiltration (Figure 6, A and B) associated with apoptosis and dedifferentiation (27). The impressive degree of injury was nearly completely reversed over a 7-day period (Figure 6C). Jensen et al. reported peak activation of Notch $1 / 2$ after 3 days and a reexpression of high levels of PDX-1, suggesting reversion of injured cells to a progenitor-like state (27). However, we again found no $\beta$-galactosidase labeling in the endocrine lineage, indicating that these cells 

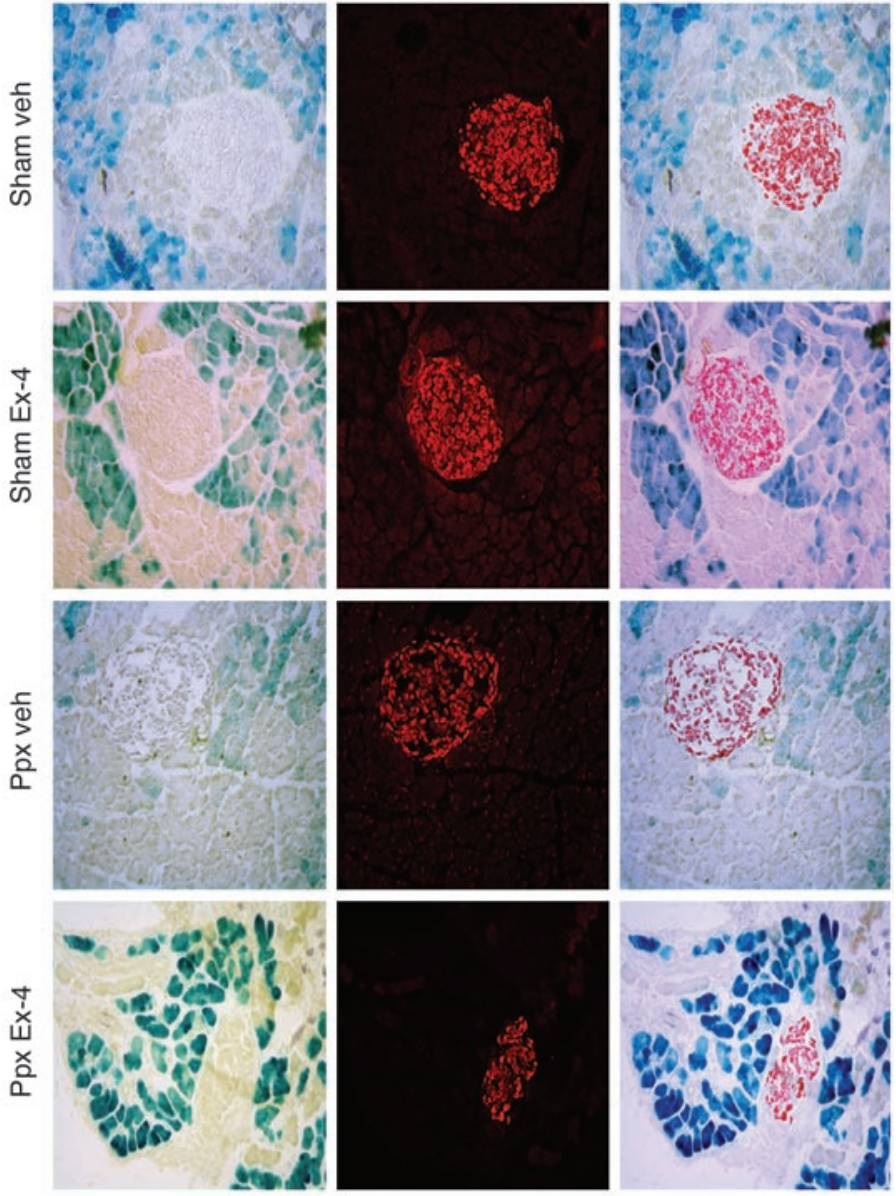

do not serve as islet progenitors even after Ex-4 administration during the peak period of Notch expression (Figure 6, D-F). In agreement with Jensen et al. (27), we did not observe labeled cells in the ductal epithelium, suggesting that acinar-to-ductal transdifferentiation did not occur in this model. Furthermore, using a lineage tracing approach similar to ours, minimal decrease of labeled acinar cells was found after recovery from cerulein-induced pancreatitis, again indicating regeneration primarily from preexisting acinar cells (J. Jensen, unpublished observations). Finally, we did not observe acinar-to-islet transdifferentiation in the pancreatic duct ligation model, although the characteristic metaplastic epithelium distal to the ligation was marked by $\beta$-galactosidase activity, indicating an acinar cell of origin (data not shown).

\section{Figure 4}

Recovery of $\beta$ cell mass after Ppx and biological efficacy of Ex-4 treatment. (A) Glucose tolerance tests were performed i.p. 2 weeks after Ppx or sham surgery in mice treated with vehicle or Ex-4 ( $n=4-5$ per group). Black line denotes sham surgery plus treatment with vehicle, blue dashed line denotes Ppx plus treatment with vehicle, gray line denotes sham surgery plus treatment with Ex-4, and dashed black line denotes Ppx plus treatment with Ex-4. $P<0.05$, Ppx plus vehicle versus all other groups; ANOVA. (B) Mass of $\beta$ cells (in mg) was determined by point counting morphometry $4-5$ weeks after surgery. (C) Weight (in $\mathrm{g}$ ) of the pancreatic remnant at $4-5$ weeks after surgery.

\section{Figure 3}

No evidence of acinar-to-islet transdifferentiation after Ppx. ElastaseCreERT2Tg/+Rosa26r+/- mice were treated with TAM for 3 weeks by subcutaneous continuous delivery pellet and subjected to $80 \%$ Ppx or sham surgery after a 1-week washout period. After surgery, mice were randomized to receive daily injections of vehicle (Veh) or Ex-4 ( $1 \mathrm{nmol} / \mathrm{kg}$ body wt i.p.; $n=4-5$ per group). Tissues were harvested 2 weeks later and stained for $\beta$-galactosidase activity (blue stain, left panels) and insulin (red stain, middle panels). Merged images are shown in the right panels. Sections depicted are from 16-week-old mice. Additional experiments carried out in mice ranging in age from 12 weeks to 6 months failed to reveal evidence of acinar-to-islet transdifferentiation. Original magnification, $\times 200$.

\section{Discussion}

To our knowledge, this is the first lineage tracing study to examine the in vivo transdifferentiation potential of pancreatic acinar cells. Our results indicate limited plasticity of differentiated acinar cells in vivo, in contrast to their apparent plasticity ex vivo, and a role for replication of preexisting acinar cells in acinar cell regeneration. The interpretation of results using this model presupposes transgene expression exclusively in fully differentiated acinar cells and not in a minor precursor cell population that may have been missed in this evaluation; however, even the latter possibility could only be reconciled with transgene activation in a progenitor cell that is fully committed to acinar cell differentiation, since no label was chased into the ductal or endocrine compartments. The particular model of injury may also influence the mechanism of the regenerative response, and it remains possible that a different injury model not considered here will reveal the potential for acinar-to-islet transdifferentiation in vivo. Finally, species specificity could lead to a different in vivo transdifferentiation potential for human acinar cells.

These results have implications for therapeutic efforts aimed at increasing insulin-producing $\beta$ cell mass for the treatment of
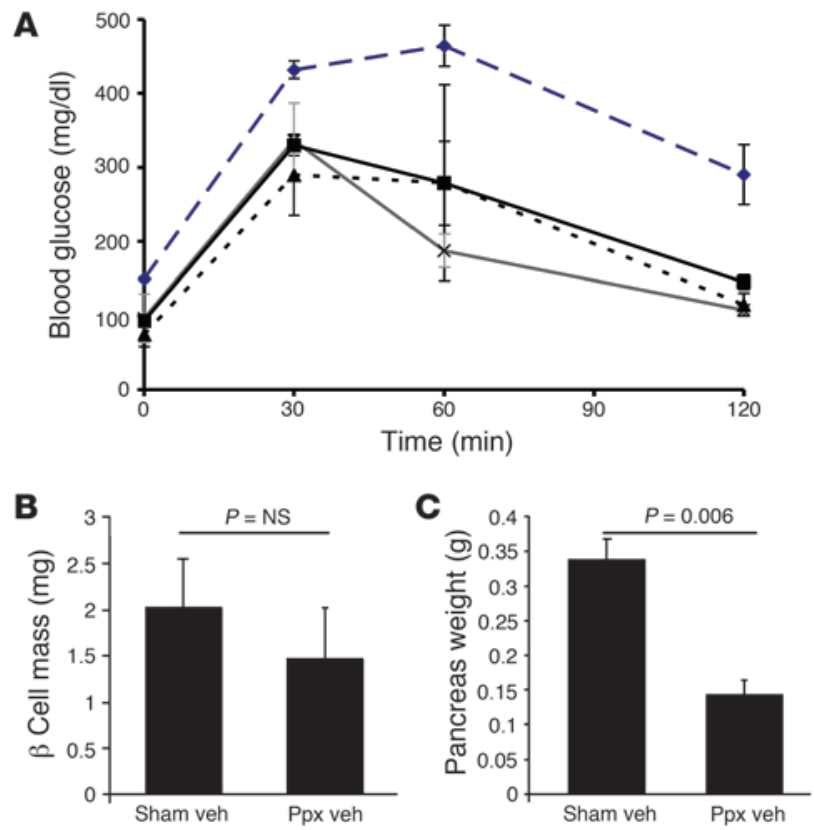


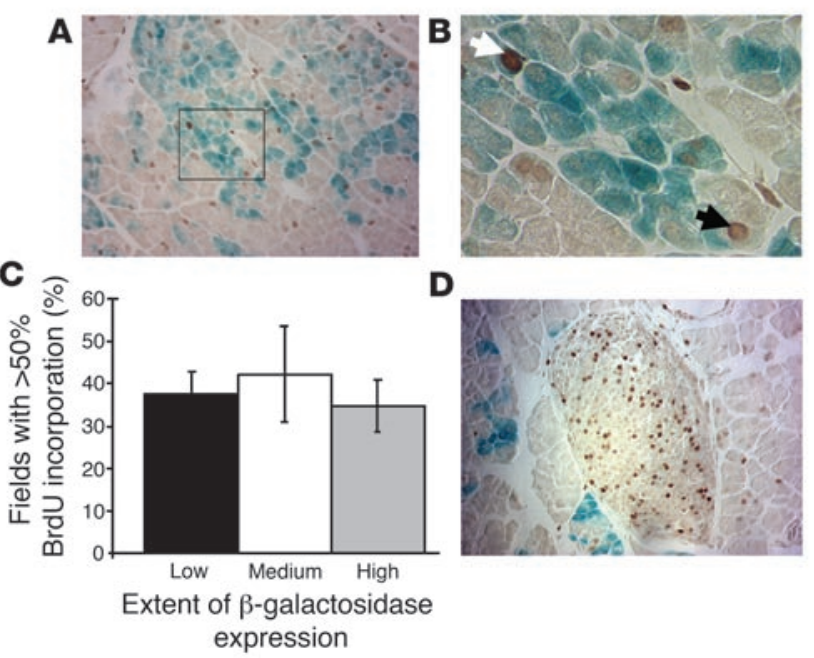

diabetes mellitus. It appears unlikely that acinar cells can change fate in vivo without additional genetic modifications, such as loss of tumor suppressor gene expression or persistent high levels of PDX-1 expression (28), or without the disruption of normal cellcell interactions as occurs during isolation and maintenance ex vivo. If these results can be applied to humans, they suggest that acinar cells in vivo may not be a suitable target for efforts to restore pancreatic $\beta$ cell mass in patients with diabetes. In contrast, recent ex vivo efforts employing acinar cells as the starting point for $\beta$ cell neogenesis have been promising (29). The lineage tracing approach in general, and the inducible Cre recombinase driver line we have developed in particular, will be useful tools for definitively proving the cell of origin in these therapeutic efforts.

\section{Methods}

Generation of the ElastaseCreERT2 transgenic mouse. The transgene was created by excising the EcoRI-EcoRI fragment containing the coding region of PDX-1 from the previously created Elastase-PDX-1 transgene (17) and replacing it with the EcoRI-EcoRI CreERT2 coding region fragment from pCreERT2 (kind gift of P. Chambon; Figure 1; ref. 30). The linearized purified insert was injected into fertilized B6SJL/F1 eggs, which were then transferred to foster females by the University of Pennsylvania Transgenic $\&$ Chimeric Mouse Facility. Offspring were genotyped by transgene-specific PCR of DNA prepared from tail biopsies taken at the time of weaning. Transgene-positive offspring were bred to $\mathrm{CD} 1$ partners. Once the line was secured, hemizygous transgenic mice were intercrossed with the

\section{Figure 6}

No evidence of acinar-to-islet transdifferentiation after cerulein-induced pancreatitis. ElastaseCreERT2Tg/+Rosa26r ${ }^{+/-}$mice were treated with TAM for 3 weeks by subcutaneous continuous delivery pellet and subjected to a 2-day course of serial cerulein injections as described in Methods. (A-C) Severe exocrine inflammation but intact islets were evident 1 day after the initiation of treatment (A and $\mathbf{B})$, with histologic recovery by 7 days $(\mathbf{C})$. Cerulein-treated mice were randomized to receive daily injections of vehicle or Ex-4 ( $1 \mathrm{nmol} / \mathrm{kg}$ body wt i.p.) on day 3 , the time of maximal Notch activation. (D-F) Tissues were harvested on day 7 , and $\beta$-galactosidase activity (blue stain) and insulin immunoreactivity (red stain) were visualized. (D) Pancreas from a control mouse that received only PBS vehicle. (E and F) Pancreata from cerulein-treated mice that received vehicle $(\mathbf{E})$ or Ex-4 (F). Original magnification, $\times 200$.

\section{Figure 5}

Regeneration of acinar cells from preexisting acinar cells after Ppx. (A) BrdU incorporation (brown stain) and $\beta$-galactosidase activity (blue stain) were assessed in ElastaseCreERT2Tg/+ Rosa2 $\mathrm{r}^{+/-}$mice that were continuously exposed to BrdU in their drinking water beginning immediately after surgery until tissue harvest 1 week later. (B) Magnification of boxed area in $\mathbf{A}$. The white arrow denotes a BrdU-positive and $\beta$-galactosidase-positive acinar cell; the black arrow denotes a BrdUpositive and $\beta$-galactosidase-negative acinar cell. (C) Percent of fields with high levels of BrdU incorporation segregated according to the proportion of acinar cells expressing $\beta$-galactosidase (low, $0 \%-30 \%$; medium, 30\%-50\%; high, 50\%-100\%). Quantitation of BrdU incorporation as a function of $\beta$-galactosidase expression was performed as described in Methods. (D) BrdU incorporation within a representative islet after Ppx. Original magnification, $\times 200$ (A and D); ×400 (B).

Rosa26r reporter mouse. All procedures were approved by the University of Pennsylvania Institutional Animal Care and Use Committee and conducted according to their guidelines.

Genotyping was carried out by PCR using primers specific for the transgene (rabbit $\beta$-globin [RBG], $5^{\prime}$-CTCTGCTAACCATGTTCATGCCT-3'; RBG455L, 5'-ACGCTAGAGCCTGTTTTG-3'; 473-bp product; annealing temperature, $60.6^{\circ} \mathrm{C}$ ) and/or LacZ (Rosa26r; LacZR, 5'-TATTTAATCAGCGACTGATCCA-3'; LacZF, 5'-TGCTGATGAAGCAGAACAACT-3'; 650-bp product; annealing temperature, $54^{\circ} \mathrm{C}$ ).

TAM administration. In the initial studies, TAM was administered to 16week-old ElastaseCreERT2 ${ }^{\mathrm{Tg} /+}{ }^{+}$Rosa26r $\mathrm{r}^{+/-}$mice according to a protocol provided by the laboratory of P. Chambon (30). Briefly, TAM (Sigma-Aldrich) was first suspended in 1 part ethanol and then mixed in 8 parts sunflower oil and stored at $-20^{\circ} \mathrm{C}$ as a $10 \mathrm{mg} / \mathrm{ml}$ solution. Mice were injected i.p. with $1 \mathrm{mg}$ TAM daily for 3-10 days. For the Ppx and other injury models, mice
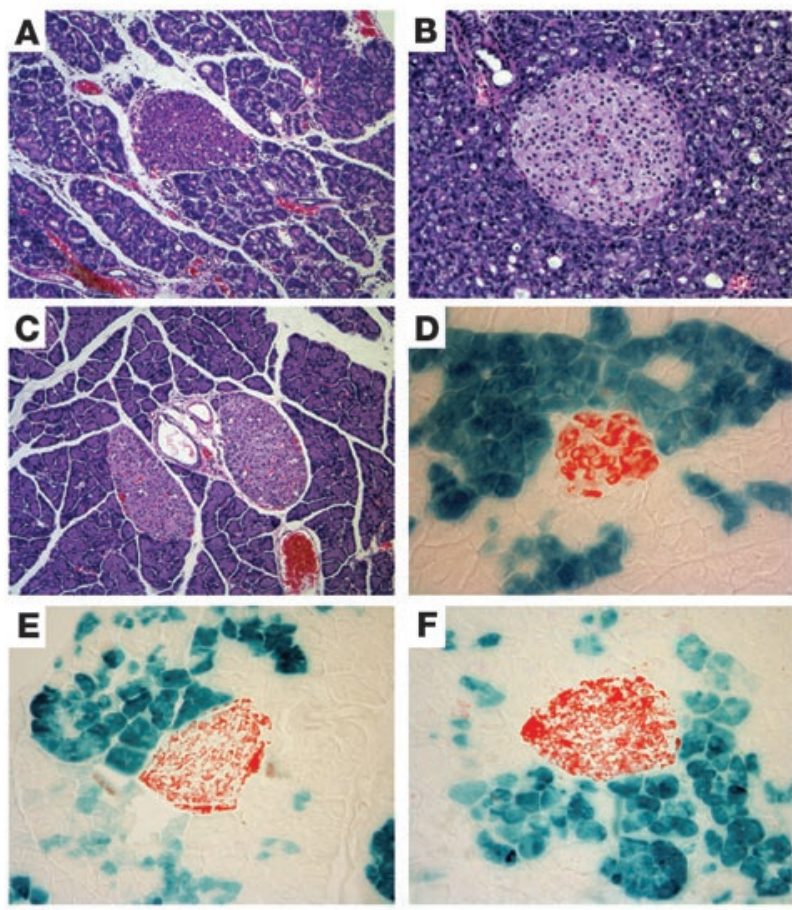
were given 1-2 mg TAM per day continuously for 21 days via a dorsally implanted subcutaneous pellet (Innovative Research of America). Previous studies have suggested that TAM is effective for 48 hours after injection (31), while another study demonstrated that CreERT2 is no longer nuclear 5 days after a 4-day course of TAM administration (P. Chambon, unpublished observations). On this basis, surgical intervention or injury model was performed 7-10 days following the termination of TAM treatment.

RNA isolation and analysis. Tissues were harvested for RNA isolation using TRIzOL reagent (Invitrogen). RNA was analyzed by RT-PCR using primers specific for Cre recombinase (RBG172, 5' - TTTTCTGAGCATACCTGG-3'; RBG455L, 5'-ACGCTAGAGCCTGTTTTG-3'; 283-bp product; annealing temperature, $60^{\circ} \mathrm{C}$ ), LacZ (described above), and HPRT mRNA (HPRTF, 5'-AGCGACAATCTACCAGAG-3'; HPRTR, 5' -AGCGCAAGTTGAATCTGC-3'; 220-bp product; annealing temperature, $60^{\circ} \mathrm{C}$ ). Reverse transcription reactions were set up using the SuperScript II RNase $\mathrm{H}^{-}$Reverse Transcriptase system (Invitrogen) and an Oligo (dT) ${ }_{15}$ Primer (Promega). Potential contamination by genomic DNA was assessed by performing PCR with an equivalent amount of RNA that did not undergo reverse transcription.

Ppx model. Mice were anesthetized by i.p. administration of Nembutal, and the abdomen was opened through an upper midline incision. We analyzed 39 mice ranging in age from 8 weeks to 6 months (specific ages for each experiment are indicated in the figure legends). The spleen and the entire splenic portion of the pancreas were excised, whereas the mesenteric pancreas between the portal vein and duodenum was left intact, resulting in a $70 \%-80 \% \mathrm{Ppx}$, as previously described (24). Sham-operated mice served as controls. The incisions were closed using 5-0 silk sutures. Ex-4 (1 nmol/ $\mathrm{kg}$ body wt dissolved in $0.9 \% \mathrm{NaCl}, 1 \% \mathrm{BSA}$ ) or vehicle was administered daily to Ppx and sham-operated mice for 10 days following surgery, starting immediately after surgery. For the final Ppx study, $\operatorname{BrdU}(1 \mathrm{mg} / \mathrm{ml})$ was administered in the drinking water of mice for 7 days starting immediately after surgery (32). Random blood glucose was measured using a handheld glucometer (FreeStyle; TheraSense). Glucose tolerance testing was carried out i.p. 12 days after surgery. Mice were injected i.p. with $2 \mathrm{~g} / \mathrm{kg}$ body wt of glucose after a 16 -hour fast.

Cerulein-induced pancreatitis model. Cerulein was administered (64 $\mu \mathrm{g}$ given as 8 hourly i.p. injections per day on days 1 and 2) in a similar manner to that previously described (27) to 6 mice, and PBS was administered to 3 control mice. On day 3 , cerulein-treated mice were additionally treated with vehicle or Ex-4 ( $1 \mathrm{nmol} / \mathrm{kg}$ body wt; $n=3$ per group). Tissues were harvested for X-gal and insulin immunofluorescence on day 8 .

Pancreatic duct ligation model. Mice $(n=9)$ were anesthetized by i.p. injection with a mixture of ketamine $(80 \mathrm{mg} / \mathrm{kg})$ and xylazine $(20 \mathrm{mg} / \mathrm{kg})$. As previously described (22), the peritoneum was entered through a midline laparotomy and the stomach, pancreas, and spleen were mobilized. The spleen was retracted laterally, and the splenic vessels were separated from the pancreatic tissue to prevent devascularization of pancreatic parenchyma. The splenic lobe of the pancreas was ligated to the left of the portal vein with a 5-0 monofilament polypropylene suture. The viscera were replaced in anatomic position, and the incision was closed in 2 layers using 4-0 braided polyglycolic acid sutures. A subcutaneous injection of $1 \mathrm{ml}$ sterile saline solution was given at the conclusion of the procedure.

Immunostaining. Tissues were harvested for immunohistochemistry at the end of the treatment period by fixing in $4 \%$ paraformaldehyde for 1 hour, cryoprotecting overnight in $30 \%$ sucrose with $1 \times$ PBS, and freezing in OCT. Frozen sections $(10 \mu \mathrm{m})$ were cut, and $\beta$-galactosidase activity was visualized by overnight incubation of frozen sections in X-gal substrate at $4^{\circ} \mathrm{C}$ using the Histomark X-Gal Kit (KPL). Slides were subsequently postfixed for 5 minutes in 4\% PFA followed by 2 washes with $1 \times$ PBS to prepare for staining. The slides were then blocked in Protein Blocking Agent (Beckman Coulter) and stained using sheep anti-BrdU (diluted 1:750; U.S. Biologicals), rabbit anti-Cre recombinase (diluted 1:50,000; Novagen), guinea pig anti-insulin (diluted 1:1,000 to 1:5,000; Linco Research Inc.), rat anti-cytokeratin 19 (diluted 1:2; Developmental Studies Hybridoma Bank, University of Iowa), biotinylated duct-specific lectin, DBA (1:50; EY Labs), or Alexa Fluor 488-conjugated (Invitrogen) DBA lectin (diluted 1:50; Sigma-Aldrich). For only BrdU staining, following postfixation and prior to blocking, all slides were pulse-microwaved for 10.5 minutes using $10 \mathrm{mM}$ citric acid buffer, $\mathrm{pH}$ 6.0. Secondary antisera include Cy3 anti-rabbit (diluted 1:1500; Jackson ImmunoResearch Laboratories Inc.), Cy2 anti-guinea pig (diluted 1:200; Jackson ImmunoResearch Laboratories Inc.) and biotinylated anti-rabbit or anti-guinea pig (1:200; Vector Laboratories). Color development for brightfield microscopy was achieved using the HRP-ABC Elite kit system and the DAB Substrate Kit for Peroxidase (Vector Laboratories).

Quantitation of $\beta$ cell mass and acinar cell proliferation. We determined $\beta$ cell mass 5 weeks after Ppx as previously described (24). A section through the maximal footprint region was stained with guinea pig anti-human insulin and visualized using 3,3'-diaminobenzidine (DAB). Sections were evaluated using a Nikon E600 microscope attached to a Photometrics CoolSnap Digital Camera. We determined $\beta$ cell mass by the point counting method using a $9 \times 10$ grid of points to assess 200 fields per pancreas. The percentage of points lying over $\beta$ cells was determined and multiplied by wet pancreas weight to determine the mass of $\beta$ cells.

Extent of acinar cell proliferation and $\beta$-galactosidase activity were assessed 1 week after Ppx and continuous administration of BrdU in the drinking water. Due to the patchy nature of $\beta$-galactosidase staining in this line, we developed a grading system to allow evaluation of entire maximal footprint cross-sections from each animal. Pancreata were harvested as previously described for immunostaining (20). A section through the maximal footprint region was first stained for $\beta$-galactosidase and subsequently stained with sheep anti-BrdU visualized using DAB. Using $0.5-\mathrm{mm}$ increments and $\mathrm{a} \times 20$ objective, fields spanning an entire maximal footprint pancreas section from each animal were captured digitally, and $\beta$-galactosidase expression and BrdU incorporation were assessed. Each captured field image was graded according to the percent of acinar cells that expressed $\beta$-galactosidase: high, $50 \%-100 \%$; moderate, $30 \%-50 \%$; low, $0 \%-30 \%$. Similarly, the percent of acinar cells showing BrdU incorporation was graded as follows: high, $50 \%-100 \%$; moderate, $30 \%-50 \%$; low, $0 \%-30 \%$. Accuracy of the grading system was confirmed in a pilot experiment in which $\beta$-galactosidase expression and BrdU incorporation were directly quantitated and correlated with estimated incorporation. Results from 4 mice were tallied to compare the distribution of highly proliferative areas within high, medium, and low $\beta$-galactosidase-positive areas.

Statistics. Values for blood glucose and $\beta$ cell mass are mean \pm SEM. Values for $\beta$ cell mass were compared using 2-tailed Student's $t$ tests. Glucose tolerance tests were compared by repeated-measures ANOVA. Differences were considered significant at $P<0.05$.

\section{Acknowledgments}

We thank Yuval Dor, Mark Magnuson, and Anil Rustgi for helpful discussions, Pierre Chambon (Institut de Genetique et de Biologie Moleculaire et Cellulaire, Cedex, France) for the CreERT2 plasmid and for the TAM administration protocol, Jake Kushner for sharing his BrdU administration protocol, Klaus Kaestner for sharing GTRosa26r mice originally provided by Philip Soriano, and David Groff and Michele Scheerer for animal husbandry support. We especially acknowledge Gary Swain, Technical Director of the Morphology Core, for help and advice with image presentation. These studies were supported by an American Diabetes Association Career Development Award (to D.A. Stoffers), NIH 
grants DK50306 (to D.A. Stoffers and S.D. Leach) and DK61215 and DK56211 (to S.D. Leach), and Individual Predoctoral NRSA 5F31HL071273 (to J. Oliver-Krasinski). This work was facilitated by the Penn Diabetes and Endocrinology Research Center (DK19525), the University of Pennsylvania Transgenic \& Chimeric Mouse Facility, and the Morphology Core of the Center for Molecular Studies in Digestive and Liver Disease (NIH grants P30 DK50306 and P01 DK49210).

1. Weir, G.C., and Bonner-Weir, S. 2004. Five stages of evolving beta-cell dysfunction during progression to diabetes. Diabetes. 53(Suppl. 3):S16-S21.

2. Naftanel, M.A., and Harlan, D.M. 2004. Pancreatic islet transplantation. PLoS Med. 1:e58; quiz e75.

3. Nanji, S.A., and Shapiro, A.M. 2006. Advances in pancreatic islet transplantation in humans. Diabetes Obes. Metab. 8:15-25.

4. Stainier, D. 2006. No stem cell is an islet (yet). N. Engl. J. Med. 354:521-523.

5. Sapir, T., et al. 2005. Cell-replacement therapy for diabetes: Generating functional insulin-producing tissue from adult human liver cells. Proc. Natl. Acad. Sci. U. S. A. 102:7964-7969.

6. Rooman, I., Heremans, Y., Heimberg, H., and Bouwens, L. 2000. Modulation of rat pancreatic acinoductal transdifferentiation and expression of PDX-1 in vitro. Diabetologia. 43:907-914.

7. Gmyr, V., et al. 2000. Adult human cytokeratin 19positive cells reexpress insulin promoter factor 1 in vitro: further evidence for pluripotent pancreatic stem cells in humans. Diabetes. 49:1671-1680.

8. Means, A.L., et al. 2005. Pancreatic epithelial plasticity mediated by acinar cell transdifferentiation and generation of nestin-positive intermediates. Development. 132:3767-3776.

9. Mashima, H., et al. 1996. Betacellulin and activin A coordinately convert amylase-secreting pancreatic AR42J cells into insulin-secreting cells. J. Clin. Invest. 97:1647-1654.

10. Shen, C.N., Slack, J.M., and Tosh, D. 2000. Molecular basis of transdifferentiation of pancreas to liver. Nat. Cell. Biol. 2:879-887.

11. Topp, B.G., McArthur, M.D., and Finegood, D.T. 2004. Metabolic adaptations to chronic glucose infusion in rats. Diabetologia. 47:1602-1610.

12. Gu, D., Arnush, M., and Sarvetnick, N. 1997. Endocrine/exocrine intermediate cells in streptozotocintreated Ins-IFN-gamma transgenic mice. Pancreas.

Received for publication August 8, 2006, and accepted in revised form January 10, 2007.

Address correspondence to: Doris A. Stoffers, Clinical Research Building, 611B, Department of Medicine, University of Pennsylvania, 415 Curie Boulevard, Philadelphia, Pennsylvania 19104-4399, USA. Phone: (215) 573-5413; Fax: (215) 898-5408; E-mail: stoffers@ mail.med.upenn.edu.

15:246-250.

13. Lardon, J., Huyens, N., Rooman, I., and Bouwens, L. 2004. Exocrine cell transdifferentiation in dexamethasone-treated rat pancreas. Virchows Arch. 444:61-65.

14. Zhou, J., Wang, X., Pineyro, M.A., and Egan, J.M. 1999. Glucagon-like peptide 1 and exendin-4 convert pancreatic AR42J cells into glucagon- and insulin-producing cells. Diabetes. 48:2358-2366.

15. Mashima, H., Shibata, H., Mine, T., and Kojima, I. 1996. Formation of insulin-producing cells from pancreatic acinar AR42J cells by hepatocyte growth factor. Endocrinology. 137:3969-3976.

16. Hammer, R.E., et al. 1987. The rat elastase I regulatory element is an enhancer that directs correct cell specificity and developmental onset of expression in transgenic mice. Mol. Cell. Biol. 7:2956-2967.

17. Heller, R.S., et al. 2001. Improved glucose tolerance and acinar dysmorphogenesis by targeted expression of transcription factor PDX-1 to the exocrine pancreas. Diabetes. 50:1553-1561.

18. Palmiter, R.D., et al. 1987. Cell lineage ablation in transgenic mice by cell-specific expression of a toxin gene. Cell. 50:435-443.

19. Soriano, P. 1999. Generalized lacZ expression with the ROSA26 Cre reporter strain. Nat. Genet. 21:70-71.

20. Gannon, M., Herrera, P.L., and Wright, C.V. 2000. Mosaic Cre-mediated recombination in pancreas using the $\mathrm{pdx}-1$ enhancer/promoter. Genesis. 26:143-144.

21. Kobayashi, H., et al. 2002. Lectin as a marker for staining and purification of embryonic pancreatic epithelium. Biochem. Biophys. Res. Commun. 293:691-697.

22. Scoggins, C.R., et al. 2000. p53-dependent acinar cell apoptosis triggers epithelial proliferation in duct-ligated murine pancreas. Am. J. Physiol. Gastrointest. Liver Physiol. 279:G827-G836.
23. Xu, G., Stoffers, D.A., Habener, J.F., and BonnerWeir, S. 1999. Exendin-4 stimulates both beta-cell replication and neogenesis, resulting in increased beta-cell mass and improved glucose tolerance in diabetic rats. Diabetes. 48:2270-2276.

24. De Leon, D.D., et al. 2003. Role of endogenous glucagon-like peptide- 1 in islet regeneration after partial pancreatectomy. Diabetes. 52:365-371.

25. Dor, Y., Brown, J., Martinez, O.I., and Melton, D.A. 2004. Adult pancreatic beta-cells are formed by self-duplication rather than stem-cell differentiation. Nature. 429:41-46.

26. Georgia, S., and Bhushan, A. 2004. Beta cell replication is the primary mechanism for maintaining postnatal beta cell mass. J. Clin. Invest. 114:963-968. doi:10.1172/JCI200422098.

27. Jensen, J.N., et al. 2005. Recapitulation of elements of embryonic development in adult mouse pancreatic regeneration. Gastroenterology. 128:728-741.

28. Miyatsuka, T., et al. 2006. Persistent expression of PDX-1 in the pancreas causes acinar-to-ductal metaplasia through Stat3 activation. Genes Dev. 20:1435-1440.

29. Minami, K., et al. 2005. Lineage tracing and characterization of insulin-secreting cells generated from adult pancreatic acinar cells. Proc. Natl. Acad. Sci. U. S. A. 102:15116-15121.

30. Indra, A.K., et al. 1999. Temporally-controlled sitespecific mutagenesis in the basal layer of the epidermis: comparison of the recombinase activity of the tamoxifen-inducible Cre-ER(T) and Cre-ER(T2) recombinases. Nucleic Acids Res. 27:4324-4327.

31. Gu, G., Dubauskaite, J., and Melton, D.A. 2002. Direct evidence for the pancreatic lineage: NGN3+ cells are islet progenitors and are distinct from duct progenitors. Development. 129:2447-2457.

32. Teta, M., Long, S.Y., Wartschow, L.M., Rankin, M.M., and Kushner, J.A. 2005. Very slow turnover of betacells in aged adult mice. Diabetes. 54:2557-2567. 